\title{
Appendiceal Malignancy: The Hidden Risks of Nonoperative Management for Acute Appendicitis
}

KRISTEN M. WESTFALL, M.P.H., ${ }^{*}$ REBECCA BROWN, M.D.,† ANTHONY G. CHARLES, M.D., M.P.H.*†

\author{
From the *University of North Carolina School of Medicine, Chapel Hill, North Carolina and +Department of \\ Surgery, University of North Carolina Hospitals, Chapel Hill, North Carolina
}

\begin{abstract}
One potential harm of nonoperative management for acute appendicitis is missed appendiceal cancer, a rare and often aggressive malignancy due to the frequency of late stage of diagnosis. Previous studies have reported an increasing incidence of appendiceal neoplasms in the population. This is a retrospective case-control study of 1007 adult patients, who presented to the University of North Carolina-Memorial Hospital (UNC-MH) between 2011 and 2015 with clinical signs and symptoms of appendicitis. We evaluated the incidence of primary appendiceal cancer in this population and determined factors that predict appendiceal cancer diagnosis using multivariate logistic regression analysis. The overall incidence of appendiceal neoplasm for adult patients presenting to UNC-MH with appendicitis from 2011 to 2015 was 2.3 per cent (23/1007). The incidence in patients without appendiceal perforation on pathology was 1.9 per cent $(16 / 832)$. Age (odds ratio (OR) 1.03), number of days of abdominal pain (OR 1.16), self-reported fever (OR 2.08), appendiceal width (OR 1.95), and appendiceal wall thickness (OR 1.30) were predictors of appendiceal neoplasm diagnosis in patients that present with acute appendicitis. We recommend that an operative approach to acute appendicitis should remain the standard of care because operative management may not only be diagnostic but potentially therapeutic.
\end{abstract}

A CUTE APPENDICITIS Is a common cause of abdominal pain in pediatric and adult patients, with a lifetime risk of 7 to 8 per cent. ${ }^{1}$ There has been a recent movement toward nonoperative management (NOM) of acute uncomplicated appendicitis. One major risk of NOM is overlooking potential cases of appendiceal cancer. Prior studies have reported an increasing incidence of appendiceal neoplasms within the population, with most recent estimates from 2009 noting an incidence of 0.97/100,000 people. ${ }^{2,3}$ Most appendiceal neoplasms are discovered incidentally after appendectomy. Of the more than 280,000 appendectomies performed in the United States each year, appendiceal tumors are discovered in approximately 0.9 to 1.4 per cent. ${ }^{4}$ Approximately, 50 per cent of patients who are diagnosed with an appendiceal tumor originally present with signs and symptoms of acute appendicitis. ${ }^{5}$ Given the recent desire for NOM of acute uncomplicated appendicitis, we sought to determine the incidence of primary appendiceal neoplasm in patients presenting with signs and symptoms of acute appendicitis.

Address correspondence and reprint requests to Kristen $\mathrm{M}$. Westfall, University of North Carolina School of Medicine, 4008 Burnett-Womack Building, CB\# 7228, Chapel Hill, NC 275997228. E-mail: Kristen_Westfall@med.unc.edu.

\begin{abstract}
Methods
A retrospective case-control study was completed by reviewing all patients at the University of North CarolinaMemorial Hospital (UNC-MH) who underwent appendectomy from January 1, 2011, to December 31, 2015. This study was approved by the UNC Institutional Review Board. The electronic medical record was reviewed to confirm appendectomy was performed after clinical suspicion of appendicitis. Only patients who were aged $\geq 18$ years at the time of appendectomy were included in the study. Variables collected included basic demographics, clinical and laboratory findings on admission, abdominal imaging modalities, operative procedure and characteristics, postoperative complications, and the histopathology report.
\end{abstract}

\section{Statistical Methods}

The distributions of the study variables of interest were analyzed across patients with and without primary appendiceal malignancy. Dichotomized variables for the highest white blood cell count and highest recorded temperature were created based on the Alvarado score. ${ }^{6}$ The incidence of primary appendiceal malignancy among patients with clinical signs appendicitis was calculated over time. Crude logistic regression analysis using a joint effects model was completed to evaluate factors associated with appendiceal malignancy. 
The likelihood ratio test was used to compare the fully adjusted and partially reduced models. Using multivariate logistic regression, the predicted values and 95 per cent confidence intervals (CIs) were calculated for the association between appendiceal wall thickness and appendiceal width with primary appendiceal malignancy. These values were used to determine a cutoff point at which appendiceal wall thickness and appendiceal width would predict primary appendiceal malignancy. Finally, a subgroup analysis of patients with uncomplicated appendicitis was completed to determine the incidence of primary appendiceal malignancy. All calculations and statistical analysis were performed in Stata Version 15 (Stata Statistical Software: Release 15. StataCorp LLC, College Station, TX).

\section{Results}

From 2011 to 2015 at UNC-MH, 1647 patients underwent appendectomy for any reason. After exclusion criteria, 1007 patients who underwent appendectomy for acute appendicitis were analyzed. Of these, 984 had no appendiceal malignancy on pathology, whereas 23 patients were diagnosed with primary appendiceal malignancy.

The incidence of primary appendiceal malignancy in adult patients who presented with signs and symptoms of acute appendicitis was 2.8 per cent $(6 / 213)$ in 2011 , 0.9 per cent $(2 / 209)$ in 2012,3 per cent $(6 / 199)$ in 2013 , 2.4 per cent $(4 / 170)$ in 2014 , and 2.3 per cent $(5 / 216)$ in 2015. The overall incidence of primary appendiceal malignancy was 2.3 per cent (23/1007) over five years. Of the patients diagnosed with primary appendiceal malignancy, neuroendocrine tumor (NET) was the most common type, followed by mixed adenoneuroendocrine carcinoma (MANEC) (Fig. 1).
Patient age and clinical symptoms of abdominal pain and fever were associated with an increased risk of primary appendiceal malignancy. For each one-year increase in age, there is a 1.03 increased odds $(95 \% \mathrm{CI}$ : $1.01,1.06)$ of being diagnosed with primary appendiceal malignancy. For each extra day that has a patient reports abdominal pain on admission to the emergency department, there is a 1.16 increased odds $(95 \%$ CI: 1.02 , 1.32) of being diagnosed with primary appendiceal malignancy. Patients who self-reported a fever at home had a 2.08 increased odds $(95 \%$ CI: $0.75,5.73)$ of being diagnosed with primary appendiceal malignancy.

The size of the appendix on pathology was also correlated with an increased risk of primary appendiceal malignancy. For each 1-cm increase in appendiceal width on pathology there was a 1.95 increased odds $(95 \% \mathrm{CI}$ : $1.16,3.26$ ) of being diagnosed with primary appendiceal malignancy. Appendiceal wall thickness was also correlated with malignancy. For each 1-mm increase in wall thickness there is a 1.30 increased odds (95\% CI: 1, 1.69) of being diagnosed with primary appendiceal malignancy.

Using multivariate logistic predictive modeling, we found that patients with an appendiceal wall thickness of $13.05 \mathrm{~mm}$ unadjusted, or $19.64 \mathrm{~mm}$ adjusted for other covariates, have a 50 per cent probability of being diagnosed with primary appendiceal malignancy on pathology. Those with an appendiceal width of $6.25 \mathrm{~cm}$ unadjusted, or $7.75 \mathrm{~cm}$ adjusted for other covariates, also have a 50 per cent probability of being diagnosed with primary appendiceal malignancy.

Because NOM has been primarily recommended for adult patients with uncomplicated (nonperforated) acute appendicitis, we completed a subgroup analysis including only patients without appendiceal perforation

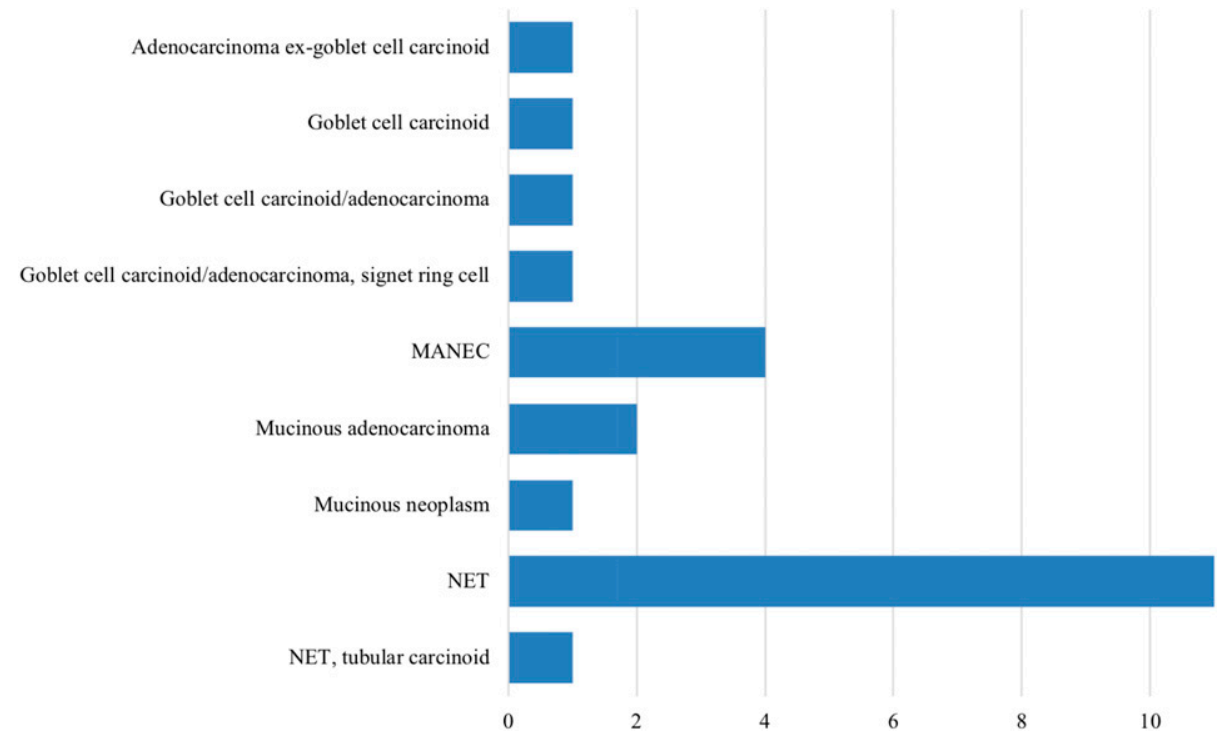

FIG. 1. Primary appendiceal malignancy type by pathology. 
on pathology. Of the 832 patients who did not have perforation, $16(1.9 \%)$ did have primary appendiceal malignancy. Although this is less than the 2.3 per cent incidence of primary appendiceal malignancy among all patients who present with acute appendicitis, this still represents a significant risk if a NOM strategy is pursued.

\section{Discussion}

As management of acute appendicitis begins to shift away from operative intervention, understanding the potential harms of NOM is critical. We determined the incidence of primary appendiceal malignancy among adult patients presenting with acute appendicitis to be 2.3 per cent over five years. The incidence of patients with uncomplicated (nonperforated) acute appendicitis, who were diagnosed with primary appendiceal malignancy, was 1.9 per cent over five years. The decision to pursue NOM in these patients may lead to significant harm, as appendiceal cancer is most often diagnosed on surgical pathology instead of imaging or clinical presentation. Given the aggressive nature of some appendiceal malignancies, the increase of NOM for treatment of acute appendicitis could lead to a later stage of diagnosis for appendiceal malignancy, and, thus increasing morbidity and mortality of appendiceal neoplasms.

The association of appendiceal diameter and wall thickness with eventual diagnosis of primary appendiceal malignancy suggests that we may be able to predict the likelihood of primary appendiceal malignancy based on the size of the appendix. Our predictive modeling demonstrates that patients with an appendix width of $6.25 \mathrm{~cm}$ or an appendiceal wall thickness of $13.05 \mathrm{~mm}$ have a 50 per cent probability of being diagnosed with primary appendiceal malignancy. We recognize that these dimensions are quite large for most patients presenting with appendicitis, likely due to the limited number of patients in our dataset $(n=23)$. Thus, a larger database of patients with primary appendiceal malignancy could be beneficial in allowing us to better understand the true association of appendiceal size and probability of primary appendiceal malignancy.

Our results, however, do show a correlation between appendiceal size and diagnosis of primary appendiceal malignancy. For example, patients with an appendiceal wall thickness of $4 \mathrm{~mm}$ have a 3.2 per cent probability of being diagnosed with appendiceal malignancy based on this imaging information alone. This can better inform risk assessment and surgical decision-making regarding appendiceal cancers in patients presenting with acute appendicitis.

One limitation of our study is that we only included patients aged 18 years or older. The use of NOM for pediatric patients with acute appendicitis may not bear the same potential risk for appendiceal malignancy as within the adult population. A key strength of our study is its large sample size $(n=1007)$ and inclusion of cases over a five-year period of time. Furthermore, the hospital at which the study was conducted (UNC$\mathrm{MH}$ ) is one of the largest hospitals in the state and serves patients from all counties and several neighboring states. Further studies are needed to corroborate these results, especially in health-care centers across the country.

\section{Conclusions}

This study is the first in which the primary outcome was to determine the incidence of primary appendiceal malignancy within a cohort of adult patients presenting with signs and symptoms of acute appendicitis. Our results demonstrate the incidence of primary appendiceal malignancy among patients presenting with signs and symptoms of acute appendicitis is approximately 2.3 per cent over five years. If a NOM strategy is pursued, the risk of delayed diagnosis with increased morbidity may be prohibitive. Operative management of acute appendicitis is not only diagnostic, but also potentially therapeutic for appendiceal cancers.

\section{REFERENCES}

1. Addiss D, Shaffer N, Fowler B, et al. The epidemiology of appendicitis and appendectomy in the United States. Am J Epidemiol 1990;132:910-25.

2. McCusker M, Coté T, Clegg L, et al. Primary malignant neoplasms of the appendix: a population-based study from the surveillance, epidemiology and end-results program, 1973-1998. Cancer 2002;94:3307-12.

3. Marmor S, Portschy P, Tuttle T, et al. The rise in appendiceal cancer incidence: 2000-2009. J Gastrointest Surg 2015;19:743-50.

4. Turaga K, Pappas S, Gamblin T. Importance of histologic subtype in the staging of appendiceal tumors. Ann Surg Oncol 2012;19:1379-85.

5. Connor S, Hanna G, Frizelle F. Appendiceal tumors retrospective clinicopathologic analysis of appendiceal tumors from 7,970 appendectomies. Dis Colon Rectum 1998;41:75-80.

6. Alvarado A. A practical score for the early diagnosis of acute appendicitis. Ann Emerg Med 1986;15:557-64. 